\section{Low Complexity User Pairing and Power Allocation Algorithm for 5G Cellular Network Non-orthogonal Multiple Access}

\section{Jianhua He and Zuoyin Tang}

\begin{abstract}
Non-orthogonal multiple access (NOMA) is a candidate technology for the 5th generation cellular networks to reduce the big gap between network capacity and fast growing traffic. It applies superposition coding in transmitters and successive interference cancellation (SIC) at the receivers to cancel intra-cell interference. The same frequency resource can be allocated simultaneously to multiple intra-cell users, holding large potentials on improving network performance. A major technica challenge of NOMA is on user pairing and power allocation (UPPA) Most of existing UPPA algorithms are based on exhaustive search with extensive computation. A fast algorithm was proposed recently but still has high computation complexity. In this paper we propose a new algorithm, which has the lowest computation complexity achievable for NOMA UPPA. The main idea is to pair the first users having the highest proportional fairness $(\mathrm{PF})$ priority coefficient with potential second users having the highest channel conditions. And a fixed power allocation strategy is adopted. Simulation results show the proposed algorithm is significantly faster than existing algorithms while NOMA throughput gain is not sacrificed.
\end{abstract}

Introduction: Recently non-orthogonal multiple access (NOMA) was proposed as a strong candidate technology for the 5th generation (5G) cellular networks. Orthogonal multiple access (OMA) has been used in the first to the forth generation cellular networks. Working on top of the OMA framework, NOMA allows simultaneous allocation of the same frequency resource to multiple users in the same cell. Intra-cell interference is cancelled at the receivers with SIC technology. Around $20 \%$ throughput gains with NOMA have been reported in literature [1,2].

A major technical obstacle to the application of NOMA is on determining if a frequency resource block should be allocated to multiple users in their cells and how to allocate the transmit power. The problem is generally called user pairing and power allocation (UPPA) for NOMA. UPPA is vital to achieve the full potentials of NOMA. However, most of existing UPPA algorithms use exhaustive search (ES) approach to find user pairs and power allocation to demonstrate the potential performance gains with NOMA, which have very high computation complexity and can result in excessive scheduling processing delay.

Recently the authors proposed a fast and efficient UPPA algorithm with largely reduced computation complexity [3], but its complexity is still high, being proportional to the number of users. In this paper we propose a new algorithm to reduce the computation complexity to the limit of UPPA algorithms. Simulation results show that the new algorithm is significantly faster than the existing ones without throughput loss. The proposed low complexity UPPA algorithm can help bring the NOMA technology closer to the real application.

System Model: A cellular network with $N_{\text {site }}$ sites equipped with one eNB each is considered. The eNBs are labelled from 1 to $N_{\text {site }}$ and eNB 1 is located at the network center. Each eNB has 3 sectors. The $j$ th sector of the $i$ th site is denoted by $\mathcal{A}_{i, j}$, where $i \in\left[1, N_{\text {site }}\right]$ and $j \in[1,3]$. Without loss of generality we can focus our analysis on the target sector $\mathcal{A}_{1,1}$.

We assume $N_{\text {ue }}$ users, which are randomly and uniformly distributed in the target sector. There are $N_{\mathrm{rb}}$ physical resource block (PRB), which represents the basic time-frequency resource unit for data transmission in LTE networks. A full buffer traffic model is assumed.

Let $\mathcal{P}_{i, j, u, r}$ denoted signal power received by a general user $u$ from a general sector $\mathcal{A}_{i, j}$ over PRB $r$, which is computed by:

$$
\mathcal{P}_{i, j, u, r}=P_{\mathrm{t}} G_{\mathrm{PL}}(i, u) G_{\mathrm{A}}(i, j, u) \psi_{i, u} \phi_{i, j, u, r} .
$$

where $P_{\mathrm{t}}$ denotes eNB transmit power over a PRB, $G_{\mathrm{PL}}(i, u)$ denotes path gain between eNB $i$ and user $u, G_{\mathrm{A}}(i, j, u)$ denotes antenna gain between sector $\mathcal{A}_{i, j}$ and $u, \psi_{i, u}$ denotes shadow fading between eNB $i$ and $u$, and $\phi_{i, j, u, r}$ denotes small scale fast fading between $\mathcal{A}_{i, j}$ and $u$ over PRB $r$.

SINR model: Under the OMA framework, a PRB (say $r$ ) is allocated to only one user (say $u$ ). The user $u$ receives no intra-cell interference. Let $\gamma_{u, r}^{\mathrm{s}}$ denote the signal to interference plus noise ratio (SINR) of user $u$ over PRB $r$ with OMA (superscript s designating OMA), computed as:

$$
\gamma_{u, r}^{\mathrm{s}}=\frac{\mathcal{P}_{1,1, u, r}}{\sum_{j=2}^{3} \mathcal{P}_{1, j, u, r}+\sum_{i=2}^{N_{\text {site }}} \sum_{j=1}^{3} \mathcal{P}_{i, j, u, r}} .
$$

As the downlink communication is assumed to be interference limited, noise power is negligible and not considered in this Letter.

Under the NOMA framework, if a PRB $r$ is allocated to only one user, the user SINR can also be denoted by $\gamma_{u, r}^{\mathrm{s}}$ and computed by (2). For simplicity, we limit the number of users that can be multiplexed over a PRB to 2. If PRB $r$ is allocated to two users (say $u_{1}$ and $u_{2}$ ), without loss of generality, we assume $\gamma_{u_{1}, r}^{s}>\gamma_{u_{2}, r}^{s}$. According to NOMA principle, at the eNB side the desired signals targeting to $u_{1}$ and $u_{2}$ are multiplexed (superimposed) over PRB $r$, with transmit power $\alpha P_{\mathrm{t}}$ and $(1-\alpha) P_{\mathrm{t}}$ for users $u_{1}$ and $u_{2}$, respectively. A necessary condition on coefficient $\alpha$ is $\alpha<0.5$, otherwise SIC at $u_{1}$ is thought to fail.

At the receiver side, user $u_{2}$ (with poorer channel condition) decodes its signal directly without SIC, by which $u_{1}$ signal is treated as intracell user interference. Let $\gamma_{u_{1}, r}^{\mathrm{m}}\left(u_{1}, u_{2}, \alpha\right)$ and $\gamma_{u_{2}, r}^{\mathrm{m}}\left(u_{1}, u_{2}, \alpha\right)$ denote SINR of users $u_{1}$ and $u_{2}$, which are multiplexed over PRB $r$ with power allocation ratio $\alpha$, respectively. The superscript $\mathrm{m}$ is designated intra-cell user multiplexing.

If $\gamma_{u_{1}, r}^{s}>\gamma_{u_{2}, r}^{s}$, we compute $\gamma_{u_{2}, r}^{\mathrm{m}}\left(u_{1}, u_{2}, \alpha\right)$ by:

$\gamma_{u_{2}, r}^{\mathrm{m}}\left(u_{1}, u_{2}, \alpha\right)=\frac{(1-\alpha) \mathcal{P}_{1,1, u_{2}, r}}{\sum_{j=2}^{3} \mathcal{P}_{1, j, u_{2}, r}+\sum_{i=2}^{N_{\text {site }}} \sum_{j=1}^{3} \mathcal{P}_{i, j, u_{2}, r}+\alpha \mathcal{P}_{1,1, u_{2}, r}}$

At user $u_{1}$ intra-cell interference from $u_{2}$ is decoded and cancelled before $u_{1}$ desired signal is decoded. SINR of user $u_{1}$ can be computed by:

$$
\gamma_{u_{1}, r}^{\mathrm{m}}\left(u_{1}, u_{2}, \alpha\right)=\frac{\alpha \mathcal{P}_{1,1, u_{1}, r}}{\sum_{j=2}^{3} \mathcal{P}_{1, j, u_{1}, r}+\sum_{i=2}^{N_{\text {site }}} \sum_{j=1}^{3} \mathcal{P}_{i, j, u_{1}, r}} .
$$

Otherwise, if $\gamma_{u_{1}, r}^{s}<=\gamma_{u_{2}, r}^{s}$, we can compute $\gamma_{u_{2}, r}^{\mathrm{m}}\left(u_{1}, u_{2}, \alpha\right)$ and $\gamma_{u_{1}, r}^{\mathrm{m}}\left(u_{1}, u_{2}, \alpha\right)$ by (4) and (3), respectively.

Scheduling and UPPA Algorithm: eNBs are responsible to allocate each PRB to one or two users in each subframe. If two users are multiplexed over a PRB, the power allocation coefficient $\alpha$ is to be determined. These decisions can be made by joint scheduling and UPPA algorithm for NOMA. In this paper we consider a general proportional fairness scheduling (PFS) algorithm. In the PFS algorithm, priority coefficient (PC) for every user over every PRB is computed. Under the OMA framework, a PRB is simply allocated to the user with the maximal PC over that PRB.

The PC of PFS algorithm is computed as the ratio of estimated instantaneous achievable throughput over a PRB to the average throughput for a user. We let $\eta_{u_{1}, r, n}\left(u_{2}, \alpha\right)$ denote the estimated instantaneous throughput of user $u_{1}$ over PRB $r$ at the $n$th subframe, in the case that $u_{1}$ and $u_{2}$ are multiplexed over PRB $r$ with a ratio $\alpha$ of transmit power allocated to the user with higher SINR. Let $\bar{\eta}_{u, n}$ denotes the average throughput of user $u$ until subframe $n$. For simplicity we use Shannon formula to compute channel capacity from SINR.

Let $F_{u_{1}, r, n}\left(u_{2}, \alpha\right)$ denote the PC of user $u_{1}$ over PRB $r$, which is shared by $u_{1}$ and $u_{2} . F_{u_{1}, r, n}\left(u_{2}, \alpha\right)$ is computed by:

$$
F_{u_{1}, r, n}\left(u_{2}, \alpha\right)=\frac{\eta_{u_{1}, r, n}\left(u_{2}, \alpha\right)}{\bar{\eta}_{u_{1}, n}}
$$

Let $\eta_{u_{1}, n}$ and $F_{u, r, n}$ denote the throughput and PF metric for user $u$ over PRB $r$ without intra-cell user multiplexing, which is a specific case of $\eta_{u_{1}, r, n}\left(u_{2}, \alpha\right)$ and $F_{u_{1}, r, n}\left(u_{2}, \alpha\right)$, with $u_{1}=u_{2}=u$ and $\alpha=0$.

The objective of the ES algorithms for NOMA is to maximize the sum of the PC of the users multiplexed over each PRB by searching over all the possible user pairing (including self paring) and power allocation options. There are $N_{\mathrm{ue}}^{2}$ user pairing options over each PRB. For each user pairing option, the achievable throughput of the paired users needs to be computed with formulae (3) and (4), and their PCs need to be computed with (5). The method requires intensive computation with complexity $\mathcal{O}\left(N_{\mathrm{ue}}^{2} N_{\mathrm{rb}}\right)$ even with fixed power allocation, which can result in excessive scheduling delay with a large number of users.

The main idea of the algorithm proposed in [3] (called Fast algorithm thereafter) is choosing user with the largest $\mathrm{PC}$ without multiplexing over 
unallocated PRBs as the first user of a pair for the PRB at which the $\mathrm{PC}$ is maximized; then the second user of the pair is found as the one maximizing the sum of PCs of paired users by searching over all users [3]. The Fast algorithm largely reduces computation but still has a high complexity of $\mathcal{O}\left(N_{\mathrm{ue}} N_{\mathrm{rb}}\right)$, which can be further reduced.

In this Letter we are motivated to propose a new UPPA algorithm, to reduce the computation complexity to its limit but does not sacrifice NOMA throughput gains. In the joint scheduling and UPPA algorithm, the first users of multiplexing pairs over the unallocated PRBs are identified with the same approach used in the Fast algorithm [3]. The new algorithm finds the second users of the multiplexing pairs differently, which are chosen as the ones that have the largest non-multiplexing SINR and ensure successful SIC (both users SINR larger than 0). If none such second user exists for a PRB, multiplexing is not applied over it. If two different users are multiplexed, power allocation ratio $a$ is set to 0.2 as done in [3], which does not reduce throughput much. The proposed algorithm is called Super Fast algorithm, having the lowest UPPA complexity of $\mathcal{O}\left(N_{\mathrm{rb}}\right)$. The proposed algorithm is presented below.

1) Compute SINR $\gamma_{u, r}^{\mathrm{s}}$ and $\eta_{u, r, n}$ at subframe $n$ for all users and PRBs.

2) Compute PC $F_{u, r, n}$ for all users over all PRBs at subframe $n$.

3) Find user $u_{1}^{*}$ and PRB $r^{*}$ which maximizes $F_{u_{1}, r, n}$ over all users $u_{1} \in\left[1, N_{\text {ue }}\right]$ and all unallocated PRBs.

- Find user $u_{2}^{*}$ which has the largest $\gamma_{u, r^{*}}^{\mathrm{s}}$ and guarantees successful SIC; If none exists, no multiplexing over $r^{*}$, i.e., $u_{2}^{*}=u_{1}^{*}$.

- Allocate PRB $r^{*}$ to user $u_{1}^{*}$ and $u_{2}^{*}$. Mark PRB $r^{*}$ as allocated.

- Update average throughput $\bar{\eta}_{u_{1}^{*}, n}$ and $\bar{\eta}_{u_{2}^{*}, n}$ of users $u_{1}^{*}$ and $u_{2}^{*}$.

- Update the PC of users $u_{1}^{*}$ and $u_{2}^{*}$ over unallocated PRBs.

4) Go back to Step 3) until all PRBs are allocated.

Experiment Setup and Simulation Results: We consider a cellular network with 19 eNBs and a hexagonal cell layout. Inter-site distance is 500 meters. The total transmit power over all PRBs (equal to $N_{\mathrm{rb}} P_{\mathrm{t}}$ ) is set to 24 watts. The model specified in [4] for outdoor line-of-sight communications is used for path loss $G_{\mathrm{PL}}(d)$ :

$$
G_{\mathrm{PL}}(d)=-34.02-22 \log _{10}(d)[\mathrm{dB}] .
$$

where $d$ is the distance between site $i$ and user $u$. Sector antenna model specified in [4] is used with maximum antenna gain of $15.5 \mathrm{dBi}$ and antenna front to back ratio of $25 \mathrm{~dB}$ [5]. Shadow fading $\psi_{i, u}$ between any eNB $i$ and user $u$ is assumed to follow a log-normal distribution with zero mean and $8 \mathrm{~dB}$ standard deviation [4]. The inter-site shadow fading correlation is 0.5 . Intra site sector shadowing is fully correlated. The small scale fast fading coefficient follows exponential distribution with mean of 1 .

We consider 5 UE/PRB settings on the number of users (or UEs) and the number of PRBs: $(2,1),(5,2),(10,3),(15,4)$ and $(20,5)$ for settings 1 to 5 , respectively. Each PRB has $180 \mathrm{kHz}$ bandwidth. For each setting we perform 20 drops of UEs to the network and run 5000 simulation snapshots for each UE drop to obtain mean network performance.

Six combined algorithm settings on scheduler (round robin and PF schedulers), multiple access technology (OMA and NOMA) and UPPA algorithm (ES, Fast and Super Fast algorithms) are evaluated and compared. Combined algorithm settings 1 to 4 use PF scheduler but different access and UPPA algorithms, which are labelled 'PF-OMA', 'PF-NOMA-ES', 'PF-NOMA-Fast', 'PF-NOMA-Super', respectively. Algorithm settings 5 to 6 use round robin (RR) scheduler, labelled 'RR-OMA' and 'RR-NOMA-ES', respectively. The average throughput computing window for PF scheduler is 20. For the RR scheduler $N_{\mathrm{rb}}$ for OMA and at most $2 N_{\mathrm{rb}}$ for NOMA are scheduled over the PRBs in each subframe in round robin order.

The performance metrics of interest include mean site throughput, UPPA simulation time, 5th tile and 95th tile throughput. Fig. 1(a) and Fig. 1(b) show site throughput and UPPA time per user drop versus UE/PRB settings. In general, site throughput and UPPA time of all algorithm settings monotonically increase with UE/PRB setting number. Algorithm settings with PF scheduler consistently produce much higher throughput than those with RR scheduler. Algorithm settings with NOMA achieve $16 \%$ throughput gain over the counterparts with OMA.

More specifically, the proposed Super Fast algorithm (algorithm setting 'PF-NOMA-Super') is much faster, with around 1/4.7 and 1/102 of the UPPA time of Fast algorithm and ES algorithm with PF scheduler at UE/PRB setting 5 (20 users), respectively. Meanwhile the proposed algorithm has identical throughput to these of ES algorithm and Fast algorithm (algorithm settings 'PF-NOMA-ES' and 'PF-NOMA-Fast'). It is noted that theoretically the Fast algorithm has a UPPA time 20 times of the proposed algorithm at UE/PRB setting 5. However, this is not reflected in Fig. 1(b) as data preprocessing time was included in the presented simulation time.

Fig. 1(c) and Fig. 1(d) present the 5th tile and 95th tile throughput, corresponding to users with good and poor channel conditions, respectively. It is observed that algorithm settings with $\mathrm{PF}$ scheduler have much higher 5th tile and 95th tile throughput than settings with RR scheduler for most UE/PRB settings. Algorithm setting 'PF-NOMASuper' has the largest 5th tile throughput, 15\% more than 'PF-NOMAES' and 'PF-NOMA-Fast', and 47\% more than 'PF-OMA' at UE/PRB setting 5. 'PF-NOMA-Super' has identical 5th tile throughput to 'PFOMA', but $10 \%$ less than 'PF-NOMA-ES' and 'PF-NOMA-Fast' at UE/PRB setting 5. These results show the throughput gain from NOMA goes mainly to the users with good channel conditions for 'PF-NOMASuper', and is distributed more evenly to all users for 'PF-NOMA-ES' and 'PF-NOMA-Fast'.

In conclusion, the proposed Super Fast algorithm has the lowest achievable complexity for UPPA, which is not affected by the number of users $N_{\text {ue }}$. It is significantly faster than the exhaustive search algorithm and the Fast algorithm [3] without network throughput loss. In our future work we will evaluate and compare the performance of the UPPA algorithms with more channel and antenna models.

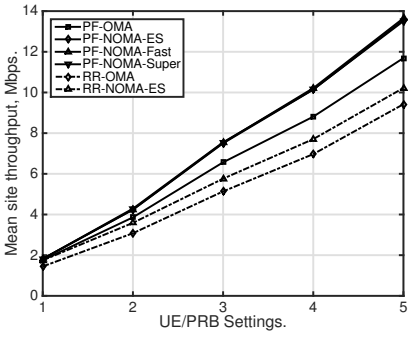

(a) Mean site throughput (Mbps)

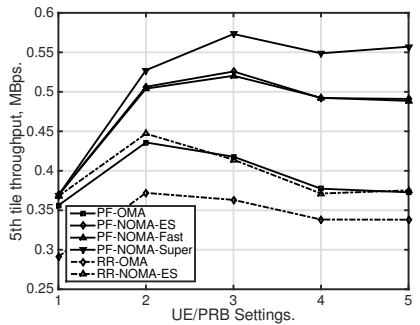

(c) 5th tile throughput (Mbps)

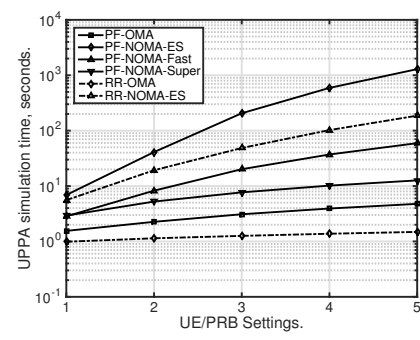

(b) Simulation time per drop

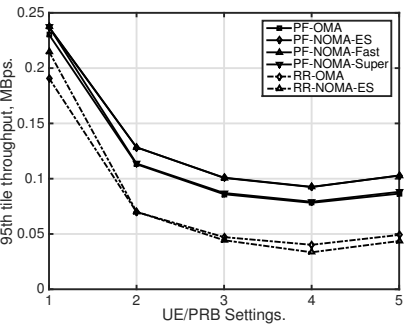

(d) 95th tile throughput (Mbps)
Fig. 1 a) Site throughput, b) UPPA simulation time, c) 5th tile throughput and d) 95th tile throughput versus UE/PRB settings.

Acknowledgment: The authors would like to acknowledge the financial support of project DETERMINE (FP7-PEOPLE-2012IRSES Proposal number: 318906)

Jianhua He and Zuoyin Tang (Aston University, School of Engineering and Applied Science, Birmingham, B4 7ET, UK). Zuoyin Tang is Corresponding Author (Email: z.tang1@aston.ac.uk).

\section{References}

1 Saito, Y., Kishiyama, Y., Benjebbour, A., et al, 'Non-orthogonal multiple access (NOMA) for cellular future radio access', IEEE VTC Spring'13, Dresden, Germany, June 2013.

2 Benjebbour, A., Li, A., Saito, Y., et al, 'System-level performance of downlink NOMA for future LET enhancements', Globecom Workshops'13, Atlanta, GA, USA, Dec. 2013.

$3 \mathrm{He}$, J., Tang, Z., Che, Z., 'Fast and efficient user pairing and power allocation algorithm for non-orthogonal multiple access in cellular networks', Electronics Letters, Nov. 2016 (in press).

4 3GPP TR 36.814 V9.0.0, 'Further advancements for E-UTRA physical layer aspects', Technical Report, March 2010.

5 He, J., Cheng, W., Tang, Z., et al, 'Analytical evaluation of higher order sectorization, frequency reuse, and user classification methods in OFDMA networks', IEEE Trans. Wireless Comm., Sept. 2016, PP, (99), pp. 1-1. 